\title{
Network-based Distributed Mobility Management for Network Mobility
}

\author{
Petro P. Ernest ${ }^{1}$, H. Anthony Chan ${ }^{2}$, Olabisi E. Falowo ${ }^{1}$, Linoh A. Magagula ${ }^{1}$, and Sandra Céspedes ${ }^{3}$ \\ ${ }^{1}$ Department of Electrical Engineering, University of Cape Town, Rondebosch 7701, South Africa \\ ${ }^{2}$ Futurewei Technologies, Plano, Texas, USA \\ ${ }^{3}$ Department of Information and Communications Technology, Icesi University, Cali, Colombia \\ Emails:prugambwa@yahoo.com; h.a.chan@ieee.org; bisi@ieee.org; linohm@yahoo.com; scespedes@icesi.edu.co
}

\begin{abstract}
Network Mobility (NEMO) Basic Support Protocol (NBSP) provides mobility supports for mobile networks. NBSP is an extension of Mobile IPv6 which employs a centralized mobility management approach. It relies on a static and centralized home agent for signaling management and data forwarding. All data traffic traverses a centralized home agent, which leads to sub-optimal routing, high packet overhead and latency, especially in nested NEMO (i.e. when the mobile networks connect to one another to reach the infrastructure). In this paper, we develop a network-based distributed mobility management (DMM) scheme for nonnested and nested NEMO scenarios, with the goal of mitigating the aforementioned problems. Additionally, the proposed scheme improves the packet delivery and location update (i.e., binding update) costs. The paper discusses in detail the scheme's design, operation mechanism and the performance evaluation analysis. The numerical results of the proposed scheme show significant improvement in packet overhead and latency as well as binding update and packet delivery costs.
\end{abstract}

Index Terms-IP mobility management, network mobility, distributed mobility management

\section{INTRODUCTION}

Nowadays, the Internet is being challenged with rapid growth in volume of mobile data traffic and increasing number of mobile users interested in accessing Internet anytime, anywhere. This is accelerated with the increase in number of smart phones, tablets and other mobile broadband capable devices. Additionally, the demand for Internet services access on moving vehicles such as cars, subways and buses is also growing.

In order to support the access of the Internet for mobile nodes in IP networks, Mobile IPv6 (MIPv6) [1] was standardized by the IETF. MIPv6 supports mobility for a single node, and allows the mobile node to remain reachable and maintain ongoing communications when the node changes the IP point of attachment to the Internet. However, MIPv6 does not support network mobility such as a group of nodes moving together in a vehicle. Consequently, the IETF has specified the Network Mobility (NEMO) Basic Support protocol (NBSP) [2] based on MIPv6, which aims to manage mobility of a moving network.

NEMO comprises one or more mobile routers. A mobile router is the default gateway of a mobile network. Packets destined to the mobile network nodes (MNNs) are encapsulated through a bi-directional tunnel established between the mobile router and its home agent (HA). When a mobile network moves to a visited network, the mobile network may connect to another mobile network and forms a nested NEMO. As the amount of nested mobile networks increases, the pinball route problem occurs [3].

Given that NBSP is based on MIPv6 operation, it employs a centralized mobility management approach that leads to problems such as [4][5]: (i) sub-optimal routing, since NBSP does not employ direct routing between an MNN and its correspondent node(s). The problem becomes severe when NEMO has multiple nested mobile networks, which leads to longer packet delivery delay and packet overhead due to the pinball routing problem; and (ii) the HA maintains the context of each mobile router and MNNs, and the traffic destined to the MNNs is first routed to the HA for packet redirection. Such a scheme has scalability issues and also leads to a single point of failure (i.e., the HA) that may lead to a service outage for a large number of MNNs. Therefore, NEMO cannot efficiently handle the increase in both number of mobile users and data traffic volume. Alternatively, mobility management based on a distributed mobility management (DMM) approach [4] [6] [7] can provide an efficient scheme for NEMO.

In this paper, the network-based DMM schemes for nonnested and nested NEMO scenarios are proposed. The schemes are developed based on the concept of decomposing the logical functions of the local mobility anchor (LMA) in Proxy MIPv6 (PMIPv6) [8] into internetwork location management (LM), home network prefix (HNP) allocation, and mobility routing (MR) functions, and then co-locating the MR to the gateway routers of different networks [9]. Moreover, the schemes extend the mobile routers with delegating router and prefix request functions [13].

The contributions of this paper are twofold: (i) to propose comprehensive schemes for non-nested and nested NEMO scenarios, which follow the concept of decomposing the LMA functions in PMIPv6, and co-locate the MR functions with the gateways in different networks, so as to mitigate the pinball routing problem and packet overhead in NEMO; and (ii) to provide a performance evaluation analysis that investigates the performance gain of the proposed scheme compared with existing centralized and distributed schemes.

The remainder of this paper is organized as follows. Section II discusses the background and related work of DMM in NEMO. Section III describes the detailed design of the proposed schemes for both non-nested and nested NEMO 
scenarios, and specifies the mobility management and handover procedures. Section IV presents the performance evaluation analysis and results. Finally, Section V concludes the paper.

\section{BACKGROUND AND RELATED WORK}

The NEMO basic support protocol (NBSP) [2] defines the mobile network as the network segment that can move and attach to an arbitrary point in the routing infrastructure. It is composed of at least one mobile router (MoR) and multiple MNNs. The MoR provides access to the MNNs for connecting to the external infrastructure, and it is in charge of the mobility management of MNNs inside the mobile network.

The NBSP requires a home agent (HA) similar to the one in MIPv6; the HA registers the mobile network's location and forwards packets to it. To achieve these functionalities, the HA performs three basic logical functions [2][9][10], (a) allocation of home address (HoA) to a mobile router (or an $\mathrm{MNN}$ ); (b) LM: managing and keeping track of internetwork location for the mobile router (or MNN), which involves the mapping of the HoA to an address where the mobile router (or MNN) is reachable; and (c) MR: intercepting packets to/from the HoA of the MNN and forwarding the packets, based on the internetwork location information from the LM, either to the destination or to some other network element that knows how to forward them to the destination. In the following paragraphs, we explain the operation of NBSP in terms of these logical functions.

Fig.1 illustrates the NBSP architecture [2] in a threenetworks scenario, namely Net1, Net2, and Net3. Net1 is the home network of mobile network1 (NEMO1), a network from which the mobile router1 (MoR1) obtains its HoA, i.e., P1::/mor1(HoA11), and a set of prefixes (also called mobile network prefix (MNP)), i.e., P11::/64, for the nodes inside NEMO1. The MoR1 uses MNP, P11::/64, to assign IP address(es) to the MNNs inside NEMO1, and this MNP remains unchanged when NEMO1 moves away from home.

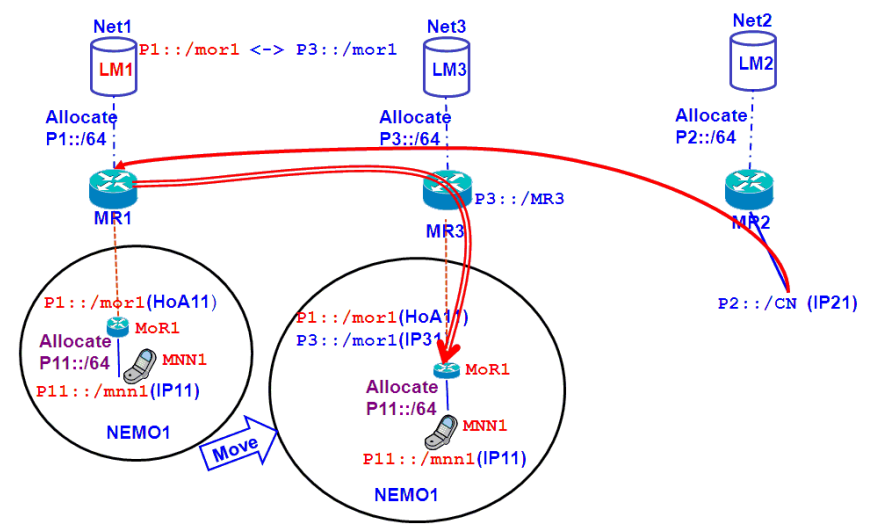

Fig. 1. NEMO Basic Support Protocol operation.

As depicted in Fig. 1, when NEMO1 moves away from home and attaches to a visited network, e.g., Net3, MoR1 configures a care-of-address (CoA) from Net3, e.g., P3::/mor1(IP31). Thereafter, MoR1 informs Net1 to update its current location, CoA, through an exchange of binding update (BU) and binding acknowledgment (BA) messages, which may also include the MNP. The LM1 of Net1 then creates a binding entry that links MoR1's HoA and MNP to the CoA. Subsequently, a bi-directional tunnel is established between MR1 and MoR1 to carry the traffic for MNNs in NEMO1. After that, if for example $\mathrm{CN}$ in Net2 sends packets destined to MNN1, they are intercepted by MR1 and then tunneled to MoR1 based on the binding information stored in LM1. Upon packets' reception and de-capsulation, MoR1 delivers the packets to MNN1. In general, all data packets from/to MNNs in NEMO1 are routed via MR1, which impose a triangular routing and tunneling overhead to each transmitted data packet.

The NBSP also considers cases in which a NEMO moves to a visited network and it attaches to another NEMO in order to reach the infrastructure. Thus, a nested mobile network is formed (i.e., nested NEMO), as shown in Fig. 2. In the figure, mobile routers MoR1, MoR4, and MoR5 represent individual NEMOs, and their home networks are Net1, Net4, and Net5, respectively. Each mobile router configures its HoA and obtains a MNP for its mobile network from their corresponding home networks. For example, MoR5 has obtained P51::/64 prefixes from Net5 to be employed as the MNP.

The mobile networks of our example move all to Net3. During the attachments, MoR1 connects to the infrastructure (i.e., MR3), MoR4 connects to MoR1, and MoR5 connects to MoR4. Hence, MoR5 configures a CoA, P41::/mor5(IP41), from MoR4's MNP. Similarly, MoR4 configures a CoA, P11::/mor4(1P31), from MoR1's MNP. As expected, MoR1 also configures a CoA, P3::/mor1(IP31), assigned by Net3. After CoA's configuration, each mobile router proceeds to notify the new location to its home network. Despite the new addressing assingments, MNNs do not perceive any changes, since mobile routers continue advertising the same MNPs..

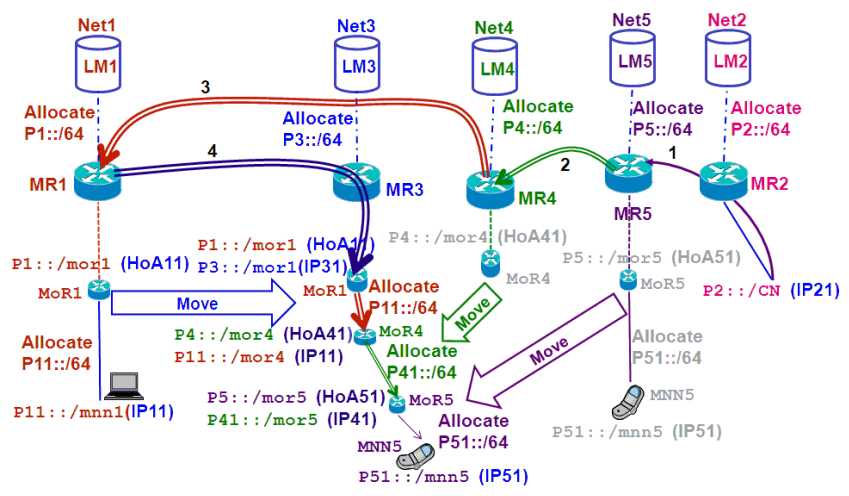

Fig. 2. Pinball routing in Nested NEMO Basic Support Protocol.

Let us assume that $\mathrm{CN}$ in Net2 sends a packet destined to MNN5 in NEMO5. The packet first visits Net5, where the IP prefix of NEMO5 is anchored (step 1). LM5 then queries the binding information for NEMO5, to find that it is attached to NEMO4. Next, MR5 encapsulates the packet with the new IP destination P41::/mor4(IP41), and tunnels it to NEMO4's home network, i.e., Net4 (step 2). The same process is repeated at Net4, where the packet are encapsulated again to be forwarded to Net1 and from there to Net3. (steps 3 and 4). At Net3, the packet is forwarded to the final destination 
through the nested NEMO. As a result of the nested scenario, the packet is forced to pass through three tunnels (steps 2 through 4), causing a large overhead due to the encapsulation process.

In addition to packet overhead, the path followed by the packet is long and sub-optimal, because of the pinball routing that appears when packets traverse all the mobile routers' home networks before reaching the final destination,. That is the effect of the centralized mobility management approach employed by NBSP,where all the mobility management logical functions are confined to the HA. Distributed Mobility Management (DMM) is one of the approaches that can address the problems identified in NBSP [5][6][7]. DMM distributes such logical functions to different networks elements while bringing them closer to the mobile nodes. Based on the concept of DMM, two DMM schemes for NEMO are proposed in [11][12].

In [11], a distributed mobility scheme based on NEMO for MIPv6 network is proposed. The scheme is developed for both non-nested and nested NEMO. The HA in MIPv6 is distributed to each access router, the default gateway of NEMO. Every time a NEMO moves to a different network, the mobile router configures a new address, registers it with the HA where the traffic is anchored, and configures a mobile network prefix from the new network. New IP sessions use the MNP assigned in the new network. However, the downside of this scheme is that the tunnel created over the wireless link for ongoing connections has negative impact on the wireless bandwidth efficiency. Moreover, the ongoing long lasting traffic may be subject to long routes because of the static traffic anchoring.

Another distributed mobility scheme to support mobile networks in vehicular scenarios and flat network architectures is proposed in [12]. The scheme employs network-based mobility, and combines the local mobility anchor (LMA) and mobile access gateway (MAG) functions of Proxy MIPv6 in each access router. The scheme introduces a proxy router to manage the MNs mobility in the mobile network and a central session database to enable reachability of the MNs and the proxy router. The MNs configure new addresses from the different networks the mobile network visits and uses the new address for new IP sessions. However, the scheme has not addressed the nested NEMO problem.

\section{PROPOSED NETWORK-BASED DMM SCHEMES FOR NEMO}

This section proposes the network-based DMM schemes for non-nested NEMO and nested NEMO scenarios. Considering the DMM requirement of reusing existing mobility protocols [4], our schemes decompose the logical functions of the LMA in PMIPv6 [8] to location management (LM), mobility routing (MR), and home network prefix (HNP) allocation functions, in a similar way to [9]; however, the scheme in [9] does not cover mobility for moving networks (NEMOs). In addition, our schemes co-locate the MR function at the gateway of each network, which in a flat network architecture, may coincide with the access router; in this way, the data-plane routing function for mobile network nodes is served by the local MR function at the network gateway.
Fig. 3 shows an example of the architecture of our nonnested NEMO DMM scheme, which comprises a large domain divided into three networks, Net1, Net2, and Net3. Each network has the mobility management functions distributed as follows: (1) an LM server, (2) an MR co-located with the gateway router $(\mathrm{GW})$, and (3) multiple access routers with mobility client functions equivalent to the MAGs in PMIPv6. Each network owns a unique IP prefix block from which it delegates a set of prefixes to the attached mobile networks. In our example, Net1 owns P1:: /64 prefixes, Net2 owns P2::/64 prefixes and Net3 owns P3::/64 prefixes. Fig. 3 shows an example of the mobile network NEMO1 attached to its home network, Net1.

Each location management server, LM1, LM2, and LM3 is co-located with the HNP allocation function, so that they delegate, upon request, a set of prefixes to the mobile router(s) attached to their networks. They also maintain the mapping between the HNP and the IP address of the MR located at the visited network where the NEMO is located. For example, if NEMO1 in Fig. 3 moves and attaches to Net2, LM1 keeps the mapping between the MoR1'S HNP and the address of MR2. Therefore, the LM servers contain a distributed database of LMs in the domain, and they can be virtually or physically deployed.
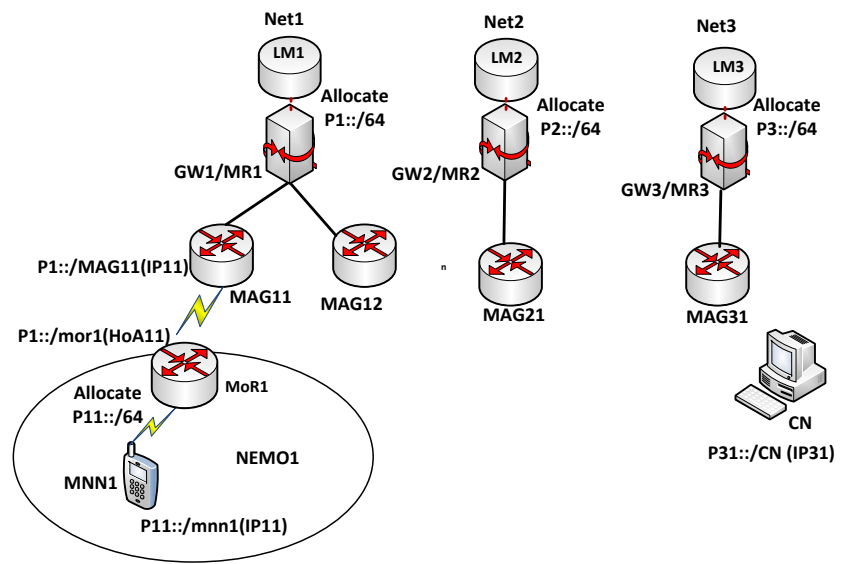

Fig. 3. A network-based DMM architecture with MR co-located at the gateways for non-nested NEMO.

On the other hand, the MR keeps the binding information between the mobile router's HNP and the address of the MAG that is currently serving the mobile router. To achieve an internetworking mobility routing, each MR interacts with the LM to retrieve the location information of the mobile router attached to its network. Moreover, the data plane routing functions for the MNNs is performed by the MR function colocated at the gateway in the visiting network. This alleviates the need for MNN's traffic to traverse the mobile router's home network, hence addressing the pinball routing problem.

Since our scheme is based on PMIPv6, which has no mobility support for mobile networks, the proposed scheme co-locates the Delegating Router (DR) function [13] with the LMs, so they can delegate IP prefixes to the mobile router(s), in order to be assigned to the MNNs. The scheme also extends the mobile router functionality with the DR function as well as with a Request Router (RR) function, as defined in [13]. The $\mathrm{RR}$ allows the mobile router to obtain a topologically-correct 
IP prefix which is then advertised to the mobile network nodes. The DR function enables the parent mobile router to delegate a subset of this topologically-correct prefixes to mobile routers attached to the mobile network, in the case of nested NEMO. Moreover, every mobile router detects the attachment of the MNNs to its access network in the same way that a MAG detects the MN attachment in PMIPv6.

Every MAG is collocated with the DHCPv6 [14] relay function, in order to handle the prefix request message sent from a mobile router. As a result, the MAGs are able to relay prefix request messages to the DR in their network. Other than the DHCPv6 relay functions, the MAGs behave in the same way as in PMIPv6, so they perform mobility management signaling on behalf of the mobile routers that attache on the access networks.

In the following subsections, we describe the detailed operation and signaling of our schemes for both non-nested and nested NEMO scenarios.

\section{A. Mobile Router Registration and Prefix Acquisition Procedure}

The signaling call flow for the attachment and registration procedures for a mobile router (MoR1) is illustrated in Fig. 4. In the figure, we use the same example illustrated in Fig. 3. When MAG11 detects MoR1's attachment, it obtains the MoR1's identifier, MoR1-id. Next, MAG11 notifies MoR1's connection to MR1, by means of a Proxy Binding Update (PBU) message. MR1 queries LM1, and the latter allocates a HNP to MoR1, i.e., P1::/64(mor1). After that, MR1 stores the mapping between the assigned prefix and MAG11's IP address, followed by the sending of a Proxy Binding Acknowledgment (PBA) to MAG11 [8]. Upon PBA's reception, MAG11 records the entry for $\mathrm{P} 1:: / 64$ (mor 1 ) and MoR1-id in its binding update list (BUL), followed by the sending of router advertisemens (RA) messages to advertise the allocated prefix to MoR1. Lastly, MoR1 uses the advertised prefix to configure its HoA, P1::/mor1(HoA11).

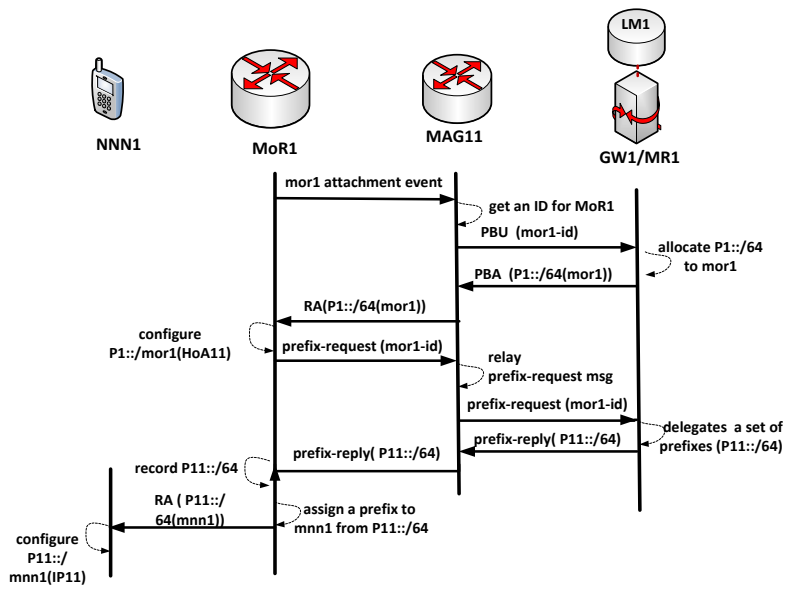

Fig.4. Mobile router registration and $\mathrm{MNN}$ attachment in DMM domain.

After MoR1 has successfully configured its home address, it runs the router request function to send a prefix request message to the DR (co-located with the LM1). Once the prefix request message is received by MAG11, it proceeds to relay the message to MR1. MR1 interacts with LM1 and the DR at LM1 allocates a prefix that is a subset of MoR1's HNP (i.e., P11::/64). Thereafter, MR1 forwards the delegated prefix to MoR1 in a prefix reply message, which is then relayed by MAG11 to MoR1. At MoR1, the prefix reply message reception triggers the creation of a prefix pool for the handling of the prefixes. At this point, MoR1 has topologically-correct prefixes to be advertised to the mobile network nodes.

When the MNN1 attaches to MoR1, MoR1 detects the attachment and allocates a prefix, P11::/64(mnn1), from its prefix pool. It also creates an entry that associates this prefix with the MNN1-id. Lastly, MoR1 sends an RA message to advertise the prefix to MNN1. Once MNN1 configures a valid IP address, it may initiate an IP session with the CN.

\section{B. Handover Mechanism for a Non-Nested NEMO Scenario}

We consider again the example of the network architecture presented in Fig.3. Let us assume that NEMO1 has moved from Net1 to Net2, while the MNN1 maintains an active communication with $\mathrm{CN}$ in Net3. This is illustrated in Fig. 5, which also shows the detailed handover operation with signaling call flow.

When NEMO1 attaches to Net2, MAG21 and MR2 perform the regular exchange of PBU/PBA messages to assign and later advertise a HNP to MoR1, i.e., P2::/64(mor1).

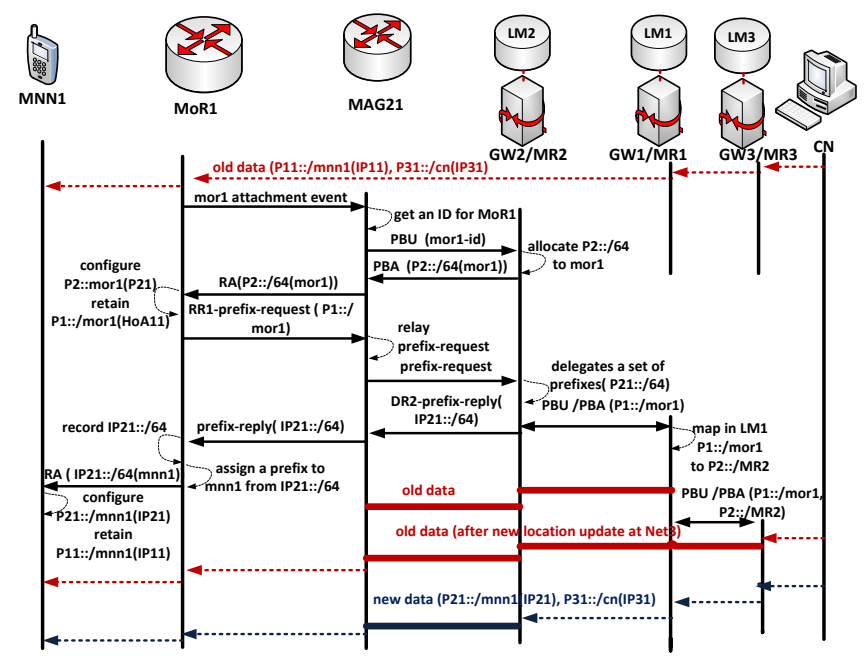

Fig.5. Mobile network handover signaling call flow for non-nested NEMO.

When MoR1 receives the RA message, it realizes there has been a change of access network; MoR1 proceeds to configure an address from the new prefix, e.g., P2::/mor1(IP21), at the same time that it keeps the old address configured from Net1, in order to maintain the ongoing session. The router request/router reply messages are also exchanged for the MoR1 to obtain prefixes for the MNNs. However, in this scenario, the prefix request message also carries the MoR1's old address. This information serves two purposes: (i) the MAG21 creates a mapping between the old 
and new addresses of MoR1, in order to allow forwarding of traffic toward MoR1 for ongoing sessions; and (ii) the MR2 learns the prefix from the network that MoR1 has detached from.

When MR2 learns such a prefix, it records the mapping between MoR1's old address and MAG21 address, so as to facilitate routing for MNNs' active session. MAG21 also interacts with LM2, in order to locate the network where MoR1's old address belongs to. Since each network owns unique IP prefixes, with the help of LMs distributed database, LM2 knows that the owner of MoR1's old prefix information is LM1, which allows MR2 to learn about MR1 for tunnel establishment. Subsequently, MR2 communicates with MR1 to establish the bi-directional tunnel that will carry the ongoing session for mobile network nodes attached to MoR1. Such a communications employs a modified PBU, which includes MR2' address, MoR1's old prefix, and an LM-flag. Upon reception, LM1 stores the mapping between MoR1's old prefix and MR2's address.

When MoR1 receives the prefix reply message from MR2, the assigned prefix is marked as new in the prefix tool, whereas the old prefix is marked as old. After that, MoR1 advertises the new prefix to MNN1 (i.e., P21::/64(mnn1)). MNN1 configures a new address from P21::/64(mnn1) but also maintains the address from the old prefix, P1::/mnn1(IP11). The new address is used to establish new IP sessions to/from MNN1, whereas the old address is used for the continuity of ongoing active sessions.

After the configuration is completed, when MR1 receives packets for MNN1 (i.e., destined to P11::/mnn1(IP11)), it retrieves the MNN1's location from LM1, which should point toward MR2 address. Thereafter, MR1 updates its routing entry with this mapping information, so that incoming packets are encapsulated to MR2, and then de-capsulated at MR2 for tunneling toward MAG21. In the same way, MAG21 decapsulates the packets and forwards them through the interface where MoR1 is attached. Thus, packets are delivered to MoR1 without tunneling over the wireless link. MoR 1 then forwards the packet to its final destination, MNN1. The routing for ongoing sessions could be further improved if MR1 informs MR3 (i.e., CN's home network) about MNN1's new location . In this way, packets destined to MNN1's old address are encapsulated from Net3 to Net2, completely bypassing Net1. This alleviates the pinball routing problem.

In the case of new IP sessions, MNN1 uses the newly configured IP address, P21::/mnn1(IP21) to establish such communications.

\section{Handover Mechanism for a Nested NEMO Scenario}

Fig.6. shows the architecture for a nested NEMO scenario with four example networks, Net1, Net2, Net3, and Net4. In the figure, NEMO1 has moved from Net1 to Net2, and NEMO4 has moved from Net4 to Net2 forming a nested NEMO (i.e., MoR4 attaches to MoR1). After NEMO1's movement, it configures an address from P2::/mor1(IP21), and obtains a set of IP prefixes, e.g., P21::/64, to be used inside the mobile network. When NEMO4 moves to Net2 and attaches to MoR1, MoR1 detects the attachment and advertises the prefix P21::/64 to MoR4. Next, MoR4 configures an IP address, e.g., P21::/mor4(IP21), while retaining its home address, P4::/mor4(HoA41).

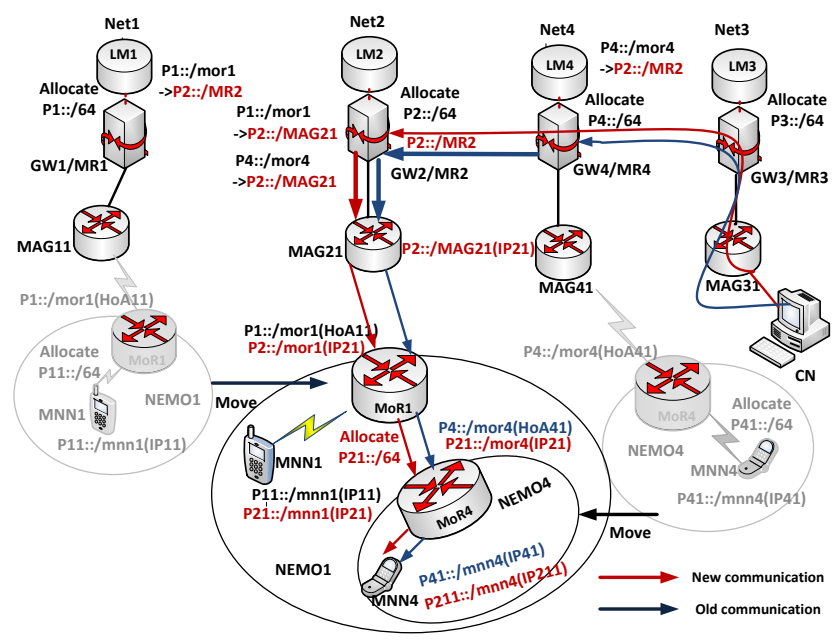

Fig. 6. A network-based DMM framework with MR co-located at the GWs for nested NEMO

After IP address configuration, MoR4 sends the prefix request message to MoR1 so as to obtain a set of prefixes for its own mobile network. The message includes MoR4' home address information, P4::/mor4(HoA41), to allow MR2 to learn about Net4 and be able to notify Net4 about the attachment of NEMO4. When MoR1 receives the prefix request message, it acts as a DR and delegates a topologicallyvalid set of prefixes to MoR4, i.e., P211::/64. Meanwhile, MoR1 also sends a modified PBU to MAG21, namely a nested PBU (nPBU), which informs Net2 about the attachment of MoR4 as a mobile network. The message includes options for MoR1's HNP, P2::/mor1(IP21), and the old address of MoR4, P4::/mor4(HoA41).

When MAG21 receives the $\mathrm{nPBU}$, it stores the mapping between $\mathrm{P} 4:: / \operatorname{mor} 4\left(\mathrm{HoA}_{41}\right)$ and $\mathrm{P} 2:: / \operatorname{mor} 1(\mathrm{P} 21)$, and then relays the message to MR2. Upong receiving the message, MR2 records the mapping between P4::/mor4(HoA41) and MAG21 address, as shown in Fig.6. With the help of LM2's distributed LMs database, MR2 locates Net4 as the owner of MoR4's address, P4::/mor4(HoA41). Thus, MR2 notifies MR4 about the new location of MoR4, and the latter requests LM4 to create an association between $\mathrm{P} 4:$ :/mor4(HoA41) and MR2's address before acknowledging to MR2. Next, MR2 responds to MoR1 with a nPBA, which confirms the successful tunnel establishmen toward Net4. The nPBA message is then relayed by MAG21 to MoR1. Upon receiving the message, MoR1 records the mapping between MoR4'S old address, P4::/mor4(HoA41), and MoR4's HNP, P21::/64(mor4).

When MoR4 receives the prefix reply message, it records the prefix in the prefix pool, as discussed in Section III.B. Such a prefix can be assigned to mobile network nodes in NEMO4. For example, MoR4 advertises P211::/64(mnn4) and MNN4 configures the address P211::/mnn4(IP211) (while keeping P41::/mnn4(IP41) for the ongoing communications). If MNN4 establishes a new IP session, it uses P211::/mnn4(IP211) so as to not traverse its home network, 
Net4. In the case of the ongoing sessions, they traverse Net4, but avoid passing thorugh Net1, as opposed to NEMO Basic Support in nested NEMO scenarios [2]. The proposed scheme may further reduce the pinball routing problem by allowing MR4 to inform MR3 about the address of MR2. Subsequently, MR3 tunnels packets for MNN4 directly to MR2; hence, packets do no longer traverse Net4, which also reduces the processing burden in the network.

\section{Performance Analysis}

In this section, the performance of the proposed schemes is analyzed and compared with NBSP [2] and NEMO-based DMM (N-DMM) [11]. N-DMM is compared to our schemes because both schemes use DMM concepts to address NEMO problems, especially for the complex nested NEMO scenario. Compared to N-DMM, our schemes decompose and distributes PMIPv6 functionality, whereas N-DMM distributes the HA of MIPv6.

In the evaluation analysis, we employ the packet overhead, end-to-end latency, packet delivery cost, and binding update cost as our performance metrics. These metrics allows us to investigate the effectiveness of the schemes in mitigating NEMO problems such as the pinball routing. Since the major focus of our proposals is to mitigate the pinball routing problem in NEMO, the analysis considers the nested NEMO scenario. We start the analysis with the nesting level presented in Fig. 6 and then extend the analysis to more than one nesting level, so as to examine the performance gain of our scheme. For comparison purposes with NBSP, HAs in NBSP are assumed to be located in the same place as MRs. Similarly, we assume that access routers (ARs) in NBSP and HAs in NDMM are positioned at the same place as MAGs in our scheme.

\section{A. Packet Overhead Analysis}

Packet overhead is defined as the amount of control information carried on a data packet because of mobility management. The additional overhead has impact on the protocol performance as it causes additional processing delay, and wastes wireless bandwidth, which is scarce and expensive. An overhead of 40 bytes per packet is used in the analysis. We consider the nested mobile network node (e.g., MNN4) to have ongoing sessions, namely the handoff traffic, before its mobile network performs handover.

Following the example shown in Fig. 6, if NBSP is employed, packets are first encapsulated by HA_Net4, and then by HA_Net1, which leads to a total of 80bytes extra overhead in the path between $\mathrm{CN}$ and MoR1. In contrast, NDMM builds a tunnel between the HA located at MAG41 and MoR4, so it incurs an extra overhead of 40bytes. The overhead of these schemes are carried over the wireless link toward MoR1. On the contrary, our scheme encapsulates packets with 40 bytes extra overhead in the wired link between MR4 and MR2, and then between MR2 and MAG41. MAG41 forwards the packet to MoR1 without any extra overhead.

With an m-level of nesting, NBSP and N-DMM present extra overhead of $40(m+1)$ bytes and 40 bytes respectively over the wireless link. In contrast, our scheme presents zero byte overhead on the wireless link. Thus, our scheme performs better in terms of packet overhead carried over the wireless link. However, our scheme incurs 40 bytes extra packet overhead in the wired link, same as N-DMM.

\section{B. End-to-end Latency Analysis}

The end-to-end latency analysis is performed for the traffic established by MNN4 before NEMO4 performs the handover to Net2. The end-to-end latency is defined as the time elapsed from when the packet is transmitted from CN's network until when it is received by MNN4. It involves the propagation delay among the various links the packet passes through. The end-to-end latency analysis for NBSP, N-DMM, and our scheme (with and without route optimization, RO), is calculated according to (1) to (4) based on the scenario presented in Fig. 6. The notations and their corresponding parameter values are presented in Table 1 .

$$
\begin{aligned}
& T_{N B S P}=t_{M R 3 / H A 3-M R 4 / H A 4}+t_{M R 4 / H A 4-M R 1 / H A 1}+t_{M R 1 / H A 1-M A G 21 / A R 21} \\
& +t_{\text {MAG21/AR21-mor } 1}+t_{\text {mor } 1-\text { mor } 4}+t_{\text {mor } 4-m m n} 4 \\
& T_{N-D M M}=t_{M A G 31 / H A 31-M A G 41 / H A 41}+t_{M A G 41 / H 41-M A G 21 / H A 21} \\
& +t_{\text {MAG21/HA21-mor } 1}+t_{\text {mor } 1-\text { mor } 4}+t_{\text {mor } 4-\text { mnn } 4} \\
& T_{\text {ours-noRO }}=t_{M R 3 / H A 3-M R 4 / H A 4}+t_{M R 4 / H A 4-M R 2 / H A 2}+t_{M R 2 / H A 2-M A G 21 / A R 21} \\
& +t_{\text {MAG21/AR21-mor } 1}+t_{\text {mor 1-mor } 4}+t_{\text {mor 4-mnn } 4} \\
& T_{\text {ours }-R O}=t_{M R 3 / H A 3-M R 2 / H A 2}+t_{M R 2 / H A 2-M A G 21 / A R 21}+
\end{aligned}
$$

\begin{tabular}{|c|c|c|}
\hline Parameter Notation & Meaning & Value \\
\hline$S_{b u}$ & BU message size (Bytes) & 72 \\
\hline$S_{p b u}^{O u}, S_{n p b u}$ & PBU message size (Bytes) & 76 \\
\hline$S_{p r m}$ & Prefix (DHCPv6) request message size (Bytes) & 96 \\
\hline$t_{H A / M R-H A / M R}, t_{H A-H A}$ & Link delay between HAs, MRs (ms) & 100 \\
\hline$t_{c n-H A / M R}$ & Link delay between $\mathrm{CN} / \mathrm{CN}$ network and $\mathrm{HA}, \mathrm{MR}(\mathrm{ms})$ & 100 \\
\hline$t_{M R / H A-A R}$ & Link delay between HA and AR (ms) & $10-100$ \\
\hline$t_{\text {mor-mor }}$ & Link delay between mors (mobile routers) (ms) & 5 \\
\hline$t_{A R-m o r}, t_{M A G-m o r}, t_{M A G / H A-m o r}$ & Link delay between MAG/AR and mor & 5 \\
\hline$t_{\text {mor-mnn }}$ & Link delay between mor and $\mathrm{mnn}(\mathrm{ms})$ & 5 \\
\hline$t_{M R-M A G}$ & Link delay between MR and MAG (ms) & 15 \\
\hline \multicolumn{2}{|c|}{$t_{M A G / A R-M A G / A R}, t_{M A G / H A-M A G / H A}$ Link delay between MAGs/ARs (ms) } & 10 \\
\hline $\begin{array}{l}p_{M R / H A}, p_{M A G}, p_{m o r} \\
m\end{array}$ & $\begin{array}{l}\text { Processing dealy of HA, MR, mor, MAG (ms) } \\
\text { Number level of nesting in nested NEMO }\end{array}$ & 10 \\
\hline
\end{tabular}

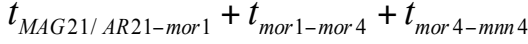

Table 1: Parameter Notations and Values [16] [17]

In order to investigate the performance gain of our scheme, in terms of the effectiveness of pinball routing mitigation, we extend the above analysis to an m-level of nesting and consider processing delays imposed by various mobility management elements the packet has to traverse. It is assumed that $\mathrm{CN}$ is sending packets to a mobile network node (MNN) located in an m-level nested mobile network. Thus, the end-to-end latency for NBSP can be expressed according to (5). N-DMM and our scheme bypass the route used by NBSP, and their endto-end latencies are given by (6), (7) and (8), respectively.

\section{Packet Delivery Cost Analysis}

As the mobile network becomes nested, the number of encapsulation grows with the increase of number of nesting level. These multiple encapsulations adds extra bits that cause additional transmission and processing costs provided by the various mobility entities traversed by the packet. The packet delivery cost (PDC) is calculated as the sum of transmission and processing costs. The transmission cost is computed as the 
Table 2: Expressions for End-to-End Latency (T), Packet Delivery Cost (PDC) and Binding Update Cost (BuC)

\begin{tabular}{|c|c|}
\hline$T_{N B S P}=\sum_{j=1}^{m+1}\left(p_{M R / H A}^{j}+p_{m o r}^{j}\right)+\sum_{j=1}^{m}\left(t_{M R / H A-M R / H A}^{j}+t_{m o r-m o r}^{j}\right)+t_{c n-M R / H A}+t_{M R / H A-A R}+t_{A R-m o r}+t_{m o r-m n n}$ & (5) \\
\hline$T_{N-D M M}=t_{c n-M A G / H A}+p_{M A G / H A}+t_{M A G / H A-M A G / H A}+t_{M A G / H A-m o r}+\sum_{j=1}^{m+1} p_{m o r}^{j}+\sum_{j=1}^{m} t_{m o r-m o r}^{j}+t_{m o r-m n n}$ & (6) \\
\hline$T_{\text {ours }-n o R O}=t_{c n-M R / H A}+2 p_{M R / H A}+t_{M R / H A-M R / H A}+t_{M R / H A-M A G}+p_{M A G}+t_{M A G-m o r}+\sum_{j=1}^{m+1} p_{m o r}^{j}+\sum_{j=1}^{m} t_{m o r-m o r}^{j}+t_{m o r-m n n}$ & (7) \\
\hline $\begin{aligned} T_{\text {ours }-R O} & =t_{c n-M A G}+t_{M A G-M R / H A}+t_{M R / H A-M R / H A}+2 p_{M R / H A}+t_{M R / H A-M A G}+p_{M A G}+t_{M A G-m o r}+\sum_{j=1}^{m+1} p_{m o r}^{j} \\
& +\sum_{j=1}^{m} t_{m o r-m o r}^{j}+t_{m o r-m n n}\end{aligned}$ & (8) \\
\hline $\begin{aligned} P D C_{N B S P} & =\sum_{j=1}^{m+1}\left(p_{M R / H A}^{j}+p_{m o r}^{j}\right)+\sum_{j=1}^{m}\left(\left(S_{\text {data }}+40 j\right) \cdot t_{M R / H A-M R / H A}^{j}+\left(S_{\text {data }}+40(m-j+1)\right) \cdot t_{m o r-m o r}^{j}\right)+t_{c n-M R / H A} \cdot S_{\text {data }} \\
& +\left(S_{\text {data }}+40(m+1)\right) \cdot\left(t_{M R / H A-A R}+t_{A R-m o r}\right)+t_{\text {mor }- \text { mnn }} \cdot S_{\text {data }}\end{aligned}$ & (9) \\
\hline $\begin{aligned} P D C_{N-D M M}= & t_{c n-M A G / H A} \cdot S_{\text {data }}+p_{M A G / H A}+\left(S_{\text {data }}+40\right) \cdot t_{M A G / H A-M A G / H A}+\left(S_{\text {data }}+40\right) \cdot t_{M A G / H A-m o r}+\sum_{j=1}^{m+1} p_{m o r}^{j} \\
& +\sum_{j=1}^{m}\left(S_{\text {data }}+40\right) \cdot t_{\text {mor-mor }}^{j}+t_{\text {mor-mnn }} \cdot S_{\text {data }} \\
\text { (10) } & \end{aligned}$ & \\
\hline $\begin{aligned} P D C_{\text {ours }-n O R O} & =t_{c n-M R / H A} \cdot S_{\text {data }}+2 p_{M R / H A}+\left(S_{\text {data }}+40\right) \cdot t_{M R / H A-M R / H A}+\left(S_{\text {data }}+40\right) \cdot t_{M R / H A-M A G} \\
& +p_{M A G}+t_{M A G-m o r} \cdot S_{\text {data }}+\sum_{j=1}^{m+1} p_{m o r}^{j}+\sum_{j=1}^{m} t_{\text {mor-mor }}^{j} \cdot S_{\text {data }}+t_{\text {mor-mnn }} \cdot S_{\text {data }}\end{aligned}$ & (11) \\
\hline $\begin{aligned} P D C_{\text {ours }-R O}= & t_{\text {cn-MAG }} \cdot S_{\text {data }}+\left(S_{\text {data }}+40\right) \cdot t_{M A G-M R / H A}+\left(S_{\text {data }}+40\right) \cdot t_{M R / H A-M R / H A}+2 p_{\text {MR/HA }} \\
& +\left(S_{\text {data }}+40\right) \cdot t_{M R / H A-M A G}+p_{M A G}+t_{M A G-m o r} \cdot S_{\text {data }}+\sum_{j=1}^{m+1} p_{m o r}^{j}+\sum_{j=1}^{m} t_{m o r-m o r}^{j} \cdot S_{\text {data }}+t_{\text {mor-mnn }} \cdot S_{\text {data }}\end{aligned}$ & \\
\hline$B u C_{N B S P}=\sum_{j=1}^{m}\left(S_{b u}+40(j-1)\right) \cdot t_{m o r-m o r}^{j}+\left(S_{b u}+40 m\right) \cdot\left(t_{M R / H A-A R}+t_{A R-m o r}\right)+\sum_{j=1}^{m}\left(S_{b u}+40(m-j)\right) \cdot t_{M R / H A-M R / H A}^{j}$ & (13) \\
\hline$B u C_{o u r s-n o R O}=S_{p r m} \cdot t_{m o r-m o r}+\sum_{j=2}^{m} S_{n p b u} \cdot t_{m o r-m o r}^{j}+S_{n p b u} \cdot t_{m o r-M A G}+S_{n p b u} \cdot t_{M A G-M R}+S_{p b u} \cdot t_{M R / H A-M R / H A}$ & (14) \\
\hline $\begin{array}{l}B u C_{o u r-R O}=S_{p r m} \cdot t_{m o r-m o r}+\sum_{j=2}^{m} S_{n p b u} \cdot t_{m o r-m o r}^{j}+S_{n p b u} \cdot t_{m o r-M A G}+S_{n p b u} \cdot t_{M A G-M R}+2 S_{p b u} \cdot t_{M R / H A-M R / H A}+S_{p b u} \cdot t_{c n-M R} \\
\text { (15) }\end{array}$ & \\
\hline$B u C_{N-D M M}=\left(t_{M A G / H A-M A G / H A}+t_{M A G / H A-m o r}\right) \cdot S_{b u}+\sum_{j=1}^{m} S_{b u} \cdot t_{m o r-m o r}^{j}$ & (16) \\
\hline
\end{tabular}

product of the data packet size and the link latency of various link types the packets traverses. The IPv6 header of 40 bytes is used in this analysis. Thus, considering the schemes' operation mechanisms and using (5) through (8), the packet delivery cost for each scheme is derived as given in (9) to (12) in Table 2.

\section{Binding Update Cost Analysis}

When a mobile network moves and attaches to a visited network through either a direct attachment or another mobile network(s), the mobile router of that network needs to send a BU message to the mobility anchor in the home network (or traffic anchoring node) so as to inform its present location.
Network resources such as transmission and processing power consumed by the $\mathrm{BU}$ messages comprise the binding update cost $(\mathrm{BuC})$. The $\mathrm{BuC}$ is defined as the product of the $\mathrm{BU}$ message size and the link delay of various link types traversed by the message. We investigate the $\mathrm{BuC}$ caused by the deepest mobile router in an m-level nested scenario. Equations (13) to (16) show the $\mathrm{BuC}$ incurred in transferring the $\mathrm{BU}$ packet from the deepest mobile router (MoR) at the m-level of nesting. In NBSP, each MoR traversed in the path from the deepest MoR toward the HA, encapsulates the packet to its corresponding $\mathrm{HA}$ with an overhead of 40 bytes. The $\mathrm{BuC}$ of the schemes is derived according to (13) to (16). 


\section{E. Numerical Results}

In this subsection, we quantitatively evaluate the performance of the proposed schemes based on the analytical functions derived in Table 2. The numerical results are generated in MATLAB using the default parameter values given in Table 1.

Fig.7 shows the numerical results that examine the impacts of different levels of nesting on the end-to-end latency of the three schemes. The resulting end-to-end latency for NBSP grows quickly because the packet traverses the HAs of parent mobile routers as given in (1) and (5). In contrast, both our scheme and N-DMM bypass the home networks of the parent mobile routers, as given in (2), (3), (4), (6), (7) and (8). Thus, both schemes achieve a smaller end-to-end latency compared to NBSP, and mitigate the effect of pinball routing. The endto-end latency for N-DMM with a link delay of $10 \mathrm{~ms}$ between HAs/ARs is slightly better than our scheme with RO. However, if the MNN has a long lasting traffic session, and its mobile network moves far away from the traffic anchoring point, as shown in Fig. 7 with a link delay of 100ms, N-DMM results in a larger end-to-end latency compared to our scheme with RO.

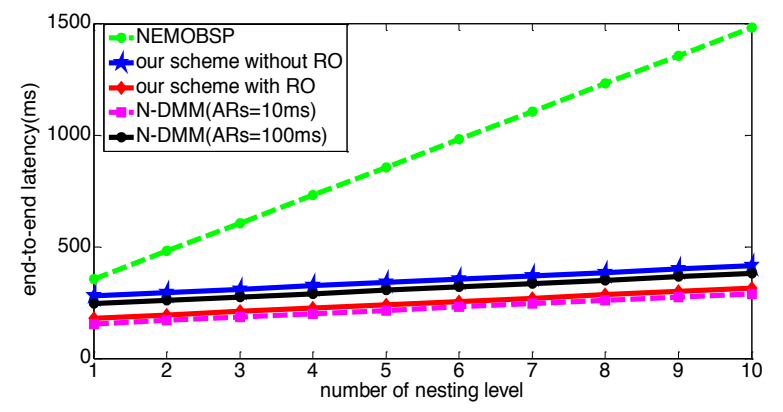

Fig. 7. End-to-end delay with different levels of nesting.

Fig.8. illustrates the trends of packet delivery cost for various levels of nesting. The data packet size $\left(\mathrm{S}_{\text {data }}\right)$ of 50 bytes is used for the calculations. It can be seen that NBSP yields a large PDC compared with other schemes because it suffers from the pinball routing problem, so the packets encounter multiple encapsulations through a long path and many processing delay. Comparing our scheme with N-DMM, our scheme has a slightly large PDC. However, when the mobile network moves far away from the traffic anchoring point, the PDC for N-DMM becomes large, as it is shown in the figure for a link delay of $100 \mathrm{~ms}$. This is due to N-DMM not optimizing the route for ongoing traffic.

Fig. 9 studies the impact of different degree of level of nesting on $\mathrm{BuC}$. The $\mathrm{BuC}$ for NBSP is large and increases very quickly compared with other schemes. This is attributed to the encapsulation of the BU messages by each mobile routers in the path from the deepest mobile router to their corresponding Has, and to the link delay between HAs. On the other hand, our RO scheme attains a slightly large $\mathrm{BuC}$ compared with $\mathrm{N}$ DMM and with our scheme without RO. This is due to additional BU message neccesary to optimize the route at the CN's network.

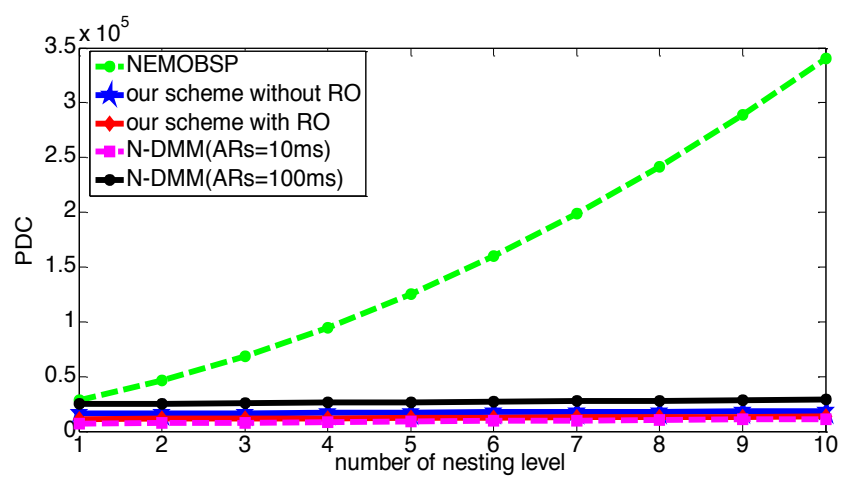

Fig. 8. Packet delivery cost with different levels of nesting.

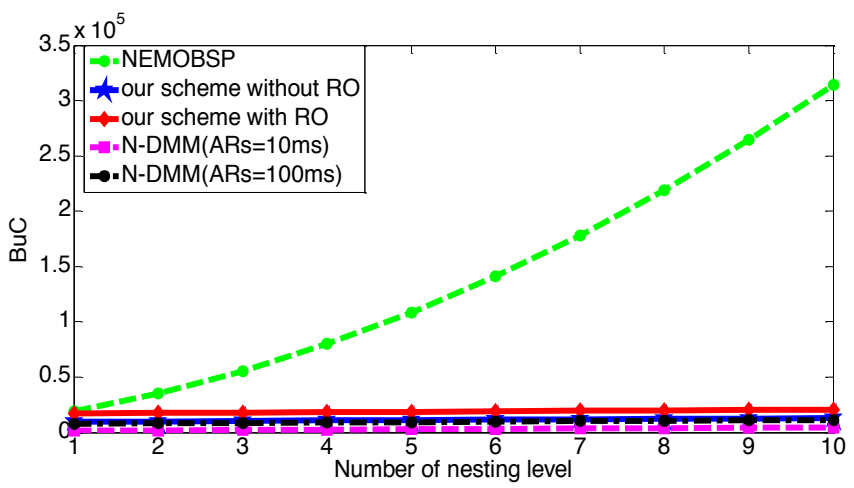

Fig. 9. Binding update cost with different levels of nesting

In addition, it can be noticed that, as the mobile network moves far away from the traffic anchoring network in $\mathrm{N}$ $\mathrm{DMM}$, the $\mathrm{BuC}$ increases as well.

\section{CONCLUSION}

In this paper we have proposed the network-based distributed schemes for both non-nested and nested NEMO scenarios. The schemes decompose the LMA entity in PMIPv6 and distributes the mobility routing function to gateways of different networks. The schemes combine this distribution with a delegating router function co-located with the location manager and the mobile routers, which helps solve the pinball routing problem in the standard NEMO Basic Support Protocol. The details of the operation of the schemes has been discussed and presented with signaling call diagram. The performance analysis demonstrates that our schemes mitigate the packet overhead and do not carries packet overhead over the wireless link. The results also show that our schemes mitigates the pinball routing problem, and achieve a reduced packet delivery latency, PDC, and BuC compared with NBSP. Although, N-DMM has a slightly smaller packet delivery delay, PDC and BuC in cases where the mobile nodes are close to anchoring network, our schemes outperforms N-DMM when the mobile network moves far away from the anchoring point. The proposed schemes are network-based DMM schemes whereas N-DMM is a host-based DMM scheme. 


\section{REFERENCES}

[1] D. Jonson, C. Perkins, and J. Arkko, "Mobility Support for IPV6," IETF RFC 6275, July, 2011

[2] V. Devarapalli, R. Wakikawa, A. Petrescu, and P. Thubert, "Network Mobility (NEMO) Basic Support Protocol," IETF RFC 3963, January 2005.

[3] T. Ernst and H. Lach, "Network Mobility Support Terminology," IETF RFC 4885, July 2007.

[4] H. Chan , D. Liu, P. Seite, H. Yokota, and J. Korhonen, "Requirements for Distributed Mobility Management" draft-ietf-dmm-requirements-03 (work in progress), December 2012.

[5] H. A. Chan, Editor, "Problem statement for distributed and dynamic mobility management," draft-chan-distributed-mobility-ps-05 (work in progress), October, 2011.

[6] P. Bertin, S. Bonjour, and J. Bonnin "Distributed or Centralized Mobility," in Proc. IEEE conference on Global telecommunications, 2009.

[7] H. A. Chan, H. Yokota, J. Xie, P. Seite, and D. Liu "Distributed and Dynamic Mobility Management in Mobile Internet: Current Approaches and Issues," Journal of Communications, vol. 6, no. 1, pp. 4-15, Feb. 2011.

[8] S. Gundavelli, K. Leung, V. Devarapalli, K. Chowdhury, and B. Patil, "Proxy Mobile IPv6," IETF RFC 5213, August,2008

[9] P. P. Ernest, H. Anthony Chan, and Olabisi E. Falowo, "Distributed mobility management scheme with mobility routing function at the gateways," GLOBECOM-2012 Wireless Networking Symposium, 2012, pp. 5476-5481.

[10]H. Anthony Chan, "Distributed mobility management with mobile IP," in Proc. International Communication Conference (ICC- Wkshps): Telecommunications: from Research to Standards, 2012, pp. 6850-6854.

[11]P. Sornlertlamvanich, S. Kamolphiwong, R. Elz and P. Pongpaibool, "NEMO-based distributed mobility management," in Proc. International Conference in Advanced Information Networking and Applications Workshops (WAINA), ,2012, pp. 645-650.

[12] Truong-Xuan Do and Younghan Kim, "Distributed network mobility management," in Proc. International Conference in Advanced Technologies for Communications (ATC), 2012, pp. 319-322.

[13] O. Troan and R. Droms, "IPv6 Prefix option for Dynamic Host Configuration Protocol (DHCP) version 6", IETF RFC 3633, December 2003

[14] R. Droms et al. "Dynamic Host Configuration Protocol for IPv6 (DHCPv6)", IETF RFC 3315, July 2003

[15] P. Bertin, S. Bonjour and J. -. Bonnin, "An evaluation of dynamic mobility anchoring," in Proc. IEEE Vehicular Technology Conference Fall (VTC 2009-Fall), 2009, pp. 1-5.

[16] M. Chuang and J. Lee, "DRO: domain-based route optimization scheme for nested mobile networks," EURASIP Journal on Wireless Communications and Networking, 2011,.

[17] Lei, J. and Fu, X. "Evaluating the Benefits of Introducing PMIPv6 for Localized Mobility Management", in Proc. IWCMC 2008, pp. 74-8. 